\title{
Processo de trabalho da equipe de enfermagem em Centros de Atenção Psicossocial Infanto-Juvenil
}

\author{
The nursing team work process in Children and Adolescents Psychosocial Care Centers \\ Proceso de trabajo del equipo de enfermería en Centros de Acompañamiento Psicosocial Infantil y Juvenil
}

Como citar este artigo:

Delfini G, Toledo VP, Garcia APRF. The nursing team work process in Children and Adolescents Psychosocial Care Centers. Rev Esc Enferm USP. 2021;55:e03775. https://doi.org/10.1590/S1980-220X2020044403775

\section{Giulia Delfini ${ }^{1}$ \\ Vanessa Pellegrino Toledo ${ }^{1}$ \\ Ana Paula Rigon Francischetti Garcia ${ }^{1}$ \\ ${ }^{1}$ Universidade Estadual de Campinas, Faculdade de Enfermagem, Campinas, SP, Brasil.}

\section{ABSTRACT}

Objective: To know the nursing team work process in Children and Adolescents Psychosocial Care Centers in a city of the state of São Paulo. Method: This is a qualitative, descriptive, and exploratory study, based on the approach of historical and dialectical materialism, carried out with the nursing team through participant observation and semi-structured interviews. Results: Eleven subjects from the nursing team participated in the study. The nursing team work process emerges from the dialectical tension of two categories: the non-knowledge, based on the alienation of the nursing work process and subordination to the knowledge of the multidisciplinary team, and the knowledge based on the nursing clinic outlined within the biological and relational perspectives. Conclusion: The nursing team work process takes place when guided by a non-knowledge resulting from alienation due to the knowledge of the other, and by knowledge through the implementation of the Nursing Process through the Nursing Care Systematization based on a biological perspective, considering relational and administrative aspects, which emerges as a contradiction.

DESCRIPTORS

Psychiatric Nursing; Nursing Process; Work; Child; Mental Health. 


\section{INTRODUÇÃO}

O movimento da Reforma Psiquiátrica (RP) no Brasil, que teve início nos anos 1970, defende a transformação do modelo assistencial em saúde mental e a construção de um novo lugar social para a loucura ${ }^{(1)}$. Como estratégia de desinstitucionalização, foram criados os Centros de Atenção Psicossocial (CAPS), que cumprem a função de oferecer cuidado intensivo a usuários com quadro psiquiátrico grave sem utilizar a hospitalização ${ }^{(1)}$. Nesse novo modelo, visando ao cuidado em saúde mental com o público infantil e juvenil, a partir de 2002 foram criados os Centros de Atenção Psicossocial Infanto-Juvenil (CAPSij) ${ }^{(1)}$.

Os CAPSij são serviços estratégicos e focados na atenção em saúde mental de crianças e adolescentes em sofrimento psíquico intenso sob o modelo de atenção psicossocial, que assume uma função que ultrapassa o fazer técnico do tratar, dando garantia à escuta, visto que ao falarem sobre si, a criança e o adolescente enxergam possibilidades de encontrar novos significados e novas formas de inserção na sociedade e família ${ }^{(2)}$. Assim, fazem parte de uma rede composta por um conjunto de serviços de saúde e de outros setores, cujo centro é o sujeito em sofrimento ${ }^{(3)}$. Porém, os CAPSij têm sido implantados de modo irregular e desigual no país, em quantidade insuficiente frente à demanda ${ }^{(3)}$.

Incrementando essa problemática, ao se considerar o campo da saúde mental infanto-juvenil na pesquisa, pouco se menciona a construção de redes de cuidado e atenção em saúde desse público ${ }^{(3)}$. Considerando o cuidado em saúde mental de crianças e adolescentes provido especificamente pela enfermagem, a produção científica é ainda mais escassa, sendo que grande parte das pesquisas abordam a relação da família com a criança que sofre transtorno mental e pouco se refere às ações de cuidado de enfermagem à criança( ${ }^{(4)}$. Além disso, os enfermeiros, técnicos e auxiliares de enfermagem não possuem disciplinas voltadas para o cuidado a esse público em sua formação ${ }^{(5)}$.

Já na prática, o processo de trabalho de enfermagem na saúde mental visa a desenvolver ações para compreender o sofrimento individual nos âmbitos psíquico, social e político, identificando as necessidades psicossociais do sujeito e superando o paradigma tutelar ${ }^{(6-7)}$. Nesse contexto, as teorias de enfermagem são fundamentais à construção do saber e prática profissional, possibilitando o desenvolvimento da tríade teoria, pesquisa e prática ${ }^{(8)}$. Tendo em vista a prática da enfermagem psiquiátrica, uma teoria amplamente utilizada é a de relação interpessoal, que acontece entre enfermeiro-paciente, pois embasa o processo de apreensão e validação das experiências do paciente, considerando para tal a forma como energia e informação fluem entre duas pessoas ${ }^{(9)}$.

Portanto, este estudo justifica-se pela produção literária escassa quando se trata do trabalho da enfermagem na assistência de crianças e jovens no campo da saúde mental no contexto brasileiro, o que contribui para uma educação acadêmica, durante a graduação, que não aborda essa especialidade do cuidado ${ }^{(5)}$, podendo resultar em profissionais despreparados e, consequentemente, em uma assistência desqualificada que pode não atender às necessidades desse público. O objetivo deste estudo é conhecer qual é o processo de trabalho da equipe de enfermagem em CAPSij de um município do estado de São Paulo.

\section{MÉTODO}

\section{TIPO DO ESTUDO}

Trata-se de um estudo qualitativo, descritivo e exploratório, embasado na abordagem do materialismo histórico e dialético, "caracterizado pelo movimento do pensamento através da materialidade histórica da vida dos homens em sociedade", descobrindo as leis fundamentais que definem sua organização ${ }^{(10)}$.

O princípio da contradição, presente nesse método, indica que para pensar a realidade é possível aceitar a contradição, caminhar por ela e apreender o que dela é essencial ${ }^{(10)}$. Nessa lógica, movimentar o pensamento significa refletir sobre a realidade partindo do empírico e, por abstrações, atingir a "compreensão mais elaborada do que há de essencial no objeto"(10).

O exercício da enfermagem requer o desenvolvimento de competências que sejam capazes de acompanhar o momento histórico e as transformações globais, visto que se insere no meio científico e tem o cuidado ao ser humano como objeto de trabalho ${ }^{(11)}$. Portanto, o método do materialismo histórico e dialético constitui um orientador de ações, ao permitir a análise histórica do processo de trabalho em enfermagem e sua vertente filosófica ${ }^{(11)}$.

\section{LOCAL}

O presente estudo foi desenvolvido em três dos quatro CAPSij localizados em Campinas, município do interior do estado de São Paulo, que possui uma Rede de Atenção Psicossocial com o objetivo de criar, ampliar e articular pontos de atenção à saúde para pessoas com sofrimento ou transtorno mental, incluindo CAPS III, CAPS Ad, CAPSij, Leitos de internação psiquiátrica em Hospital Geral, dentre outros ${ }^{(2)}$.

\section{CRITÉRIOS DE SELEÇÃo}

Os sujeitos da pesquisa foram todos aqueles que integravam as equipes de enfermagem dos três CAPSij estudados, que são compostas por enfermeiros, técnicos e auxiliares de enfermagem. Os critérios de inclusão utilizados foram: fazer parte da equipe de enfermagem de um dos CAPSij, trabalhar no serviço por um ano ou mais, e estar presente no período da coleta de dados.

\section{Coleta de Dados}

As estratégias para coleta de dados, realizada de novembro de 2019 a janeiro de 2020, foram a observação participante e a entrevista. A observação participante possibilita a imersão no cenário de pesquisa, sendo adotada a modalidade de participação ativa, que permite a movimentação do pesquisador, facilitando a observação em detalhes e profundidade em diferentes situações ${ }^{(12)}$. Esta foi realizada pelo pesquisador principal, totalizando 150 horas de observação distribuídas nos três CAPSij estudados. Já a entrevista possibilita explorar, de maneira aprofundada, as experiências dos entrevistados, 
permitindo a compreensão de como os diferentes fenômenos de interesse são experimentados e percebidos ${ }^{(13)}$. Estas foram realizadas pelo pesquisador principal no ambiente de trabalho dos participantes, em espaços privados durante as pausas dos plantões, com duração média de 23 minutos.

As entrevistas seguiram um roteiro semiestruturado, possibilitando a exploração das questões apresentadas pelo entrevistado $^{(13)}$, com as seguintes questões norteadoras: "Me conte qual é o trabalho que você desenvolve neste CAPSij", "Escolha um dia do seu trabalho e me conte suas tarefas" e "Como é seu trabalho junto às crianças com transtornos mentais?”. As entrevistas foram gravadas em áudio digital e posteriormente transcritas, sendo analisadas em conjunto com anotações das impressões do pesquisador registradas em diário de campo. $\mathrm{O}$ anonimato dos participantes foi mantido pela codificação com os termos "AE" para auxiliar de enfermagem, "TE" para técnico de enfermagem e "E" para enfermeiro, referente aos depoimentos a partir das entrevistas individuais, seguidos de algarismo arábico.

\section{ANÁlise E TRATAMENTO DOS DADOS}

A análise dos dados foi realizada a partir da análise do conteúdo, processo de descrição de dados qualitativos para representar grupos de respostas por meio do estabelecimento de categorias e identificação da frequência com que ocorrem. Em conjunto, foi utilizada a análise temática (AT), processo de interpretação de dados qualitativos, com o objetivo de encontrar padrões de significado entre os dados ${ }^{(14)}$.

A AT segue os seguintes passos para sua execução: 1 . Familiarização com os dados, mantendo contato exaustivo com o material, e formulação de códigos iniciais. 2. Agrupamento dos códigos gerados em ideias relacionadas, de forma que todos os dados relevantes sejam associados primeiro aos códigos individuais e depois aos temas principais. 3. Após identificação dos temas, estes devem ser nomeados e ilustrados com o uso de dados que capturam sua essência. 4. Exploração da relação dos temas entre si e com o contexto em que emergiram, mudando da simples descrição dos dados para seus significados, sendo necessária a situação na literatura existente sobre o assunto ${ }^{(14)}$.

\section{Aspectos ÉtIcos}

O projeto de pesquisa foi submetido ao Comitê de Ética em Pesquisa da Universidade Estadual de Campinas e aprovado sob o parecer $n^{\circ} 3.646 .481 / 2019$. A coleta de dados foi feita mediante assinatura do Termo de Consentimento Livre e Esclarecido pelos entrevistados, respeitando os princípios éticos descritos na Resolução no 466/2012, que aprova diretrizes e normas regulamentadoras de pesquisa envolvendo seres humanos.

\section{RESULTADOS}

Participaram da pesquisa 11 sujeitos da equipe de enfermagem, sendo dois auxiliares, cinco técnicos e quatro enfermeiros, totalizando duas entrevistas no primeiro CAPSij, três no segundo e seis no terceiro. $\mathrm{O}$ processo de trabalho desses profissionais nos CAPSij emerge da tensão dialética de duas categorias, apresentadas a seguir.
O NÃO SABER: ALIENAÇÃO DO PROCESSO DE TRABALHO DE ENFERMAGEM E SUBORDINAÇÃO AO SABER DA EQUIPE MULTIPROFISSIONAL

Por meio das falas, é possível identificar que os profissionais evidenciam um descompasso entre o que aprenderam em sua formação e o que realizam no serviço. Além disso, não demarcam com clareza o que a enfermagem faz no CAPSij, posto que o que reconhecem como trabalho, como a administração de medicação em pacientes, não é realizado com frequência.

Como auxiliar de enfermagem a gente faz bem pouca atividade conforme a gente aprendeu no nosso curso como auxiliar e técnico de enfermagem (AE1).

O que a enfermagem faz no CAPS? É um papel muito, muito complicado. [...] Na mental é muito mais o seu interno que você coloca (AE2).

Da questão do núcleo mesmo fica muito pouco, [...] a demanda do CAPS é muito baixa pra questões de enfermagem, de medicar, de tá fazendo um cuidado (TE1).

Também foi possível observar como o trabalho da enfermagem nos CAPSij é delimitado pelo saber do outro, que conduz o atendimento. Verifica-se que a equipe denomina seu lugar como coterapeutas, tornando seu fazer dependente de outros profissionais.

Eu deixo mais os terapeutas atuarem, fazerem o atendimento com o paciente (TE2).

Então eles falam que nós somos coterapeutas. E dai a gente entra nos grupos e meio que auxilia eles, de acordo com o foco do grupo, de cada caso (TE3).

Considerando o trabalho em equipe no campo da saúde mental, é possível observar que os profissionais de enfermagem esperam um apontamento de outros trabalhadores da equipe para desenvolver seu trabalho.

E se caso, junto com o terapeuta, junto com a psiquiatra, eles acham que precisa fazer alguma intervenção medicamentosa, dai eles acionam a gente, e a gente faz a preparação da medicação e faz a administração ali no local, mas tudo à base de conversa (TE2).

Os profissionais de enfermagem também mencionam a presença de um posto de enfermagem no serviço, além da construção da Sistematização da Assistência de Enfermagem (SAE) dos pacientes, porém como normas a serem seguidas devido a fiscalização externa.

A gente tem o posto, ele precisa tá dentro dos padrões que o Coren [Conselho Regional de Enfermagem] exige (TE4).

O Coren já esteve aqui numa fiscalização, tem que ter $100 \%$ dos usuários com $S A E(\mathrm{E} 1)$.

\section{O SABER: A CLÍNICA DE ENFERMAGEM DELINEADA ENTRE A} PERSPECTIVA BIOLÓGICA E RELACIONAL

Os achados dessa categoria mostram que o saber da enfermagem se dá pelo desenvolvimento do Processo de Enfermagem (PE) no acolhimento e consulta de enfermagem, por meio da SAE. É possível observar uma preocupação 
dos profissionais em estabelecer conversa e proporcionar bem-estar aos pacientes por meio das atividades.

A consulta de enfermagem, a gente aproveita pra fazer no acolhimento, faz aquelas perguntas sobre histórico, antecedentes familiares, pessoais, e vai tirando o diagnóstico, [...] depois tem um instrumento da nossa $S A E$ e a gente completa ele através do atendimento $(\mathrm{E} 2)$.

Então é o papel da enfermagem [...] essa parte de conversar, de ver, de se aproximar, de oferecer um bem-estar, de propor atividades [...] de um jogo pra tentar espairecer um pouquinho, $e$ através daquela atividade a gente consegue acessar um pouquinho melhor, puxar conversa, saber: e ai, como que foi o final de semana? (TE4).

No entanto, no saber da equipe de enfermagem, a SAE em alguns casos é realizada pela perspectiva biológica, reconhecendo no corpo o local do cuidado, aproximando-o daquele desenvolvido no hospital.

Uma SAE, geralmente num hospital, você vai aferir a pressão, vai ver se dormiu bem, como tá a evolução, se se alimentou bem. No nosso caso, nós temos poucas crianças hoje em dia que passam o dia aqui, então não tem muito como a gente ter esse parâmetro. [...] Semana passada foi o W. que foi medicado, então talvez nesse dia tenha uma $S A E$, mas ai vai demorar, por um lado que bom que não temos crises todos os dias, porque uma SAE num hospital é feita em todos os plantões (TE5).

Os profissionais de enfermagem, ao descreverem seu processo de trabalho, apontam situações de manejo, avaliação do comportamento, queixa da criança e atividade em grupo considerando o diagnóstico psiquiátrico.

Eu não fico só na questão da escuta, [...] eu também dou um norte. E tento trabalhar as questóes mais comportamentais, que muitas delas apresentam. São de manejo, de encaminhamento de conduta (E3).

Eu acho que a minha função é tentar ver uma relação de comportamento e de queixa, pra ver se faz sentido, tentar entender o sofrimento, tentar também pensando na queixa da criança ou do adolescente, o que a gente enquanto serviço vai conseguir ofertar (E4).

Nos grupos, depende da proposta da atividade e do grupo, então a gente faz alguma coisa mais circunscrita pra algumas crianças que têm algum déficit de atenção e hiperatividade, então de leitura e de escrita, alguns que tem uma questão mais de opositor, mais agressivo, então a gente ajuda nesse manejo, elabora algumas atividades (E2).

Também foi possível observar que os profissionais de enfermagem reconhecem seu trabalho quando atuam em situações de crise que demarcam uma intervenção sobre o corpo, e articulam a clínica à perspectiva biológica.

O que tem claro como papel eu acho que exclusivo do enfermeiro [...] é a assistência ao paciente em situações de crise, a gente precisa realizar a contenção química e física. No CAPS a participação do enfermeiro, acho que é indispensável quando acontece alguma situação clínica (E4).

Ainda ao falar do trabalho de enfermagem, os profissionais descrevem a elaboração de escala, procedimentos técnicos e supervisão da equipe de enfermagem. Apontam como característica do trabalho a liderança do plantão.

A gente faz escala de plantão, [...] se tiver alguma administração de medicação a gente realiza, [...] se tiver algum curativo a gente também faz, a assistência ao banho (TE2).

Faço a supervisão deles (E3).

É uma coisa que fica pra gente, a liderança do plantão. [...] Então vamos supor algum pedido de vaga, [...] é sempre direcionada pro enfermeiro (E4).

Por fim, é possível observar como os profissionais de enfermagem identificam seu trabalho como potente, construído a partir da discussão de seu olhar, sentimentos e percepção sobre o caso. Além disso, o trabalho é delimitado como um espaço de troca, no qual o profissional aponta seu enriquecimento em atendimentos grupais ou individuais.

Eu acho que a enfermagem é muito potente dentro do serviço de saúde mental, é sempre a maior equipe (E4).

A equipe se reúne numa reunião de todos, independente de ser 0 técnico de enfermagem, todos podem falar da mesma forma do seu olhar, da forma que viu o caso, o que que presenciou, [...] contando o que ouviu ou sentiu do caso, a percep̧ão que teve (TE5).

Os atendimentos me enriquecem, eu tô super satisfeita. Participar de grupo de familia, e fazer o atendimento individual, seja com o paciente ou com o familiar, pra mim me enriquece e eu gosto de fazer (AE2).

\section{DISCUSSÃO}

O descompasso entre o que os profissionais aprenderam em sua formação e o que realizam no serviço pode ser corroborado pelo processo histórico da educação de profissionais de saúde, baseado em critérios que enfatizam aspectos biológicos, a fragmentação do saber, e o fortalecimento da dicotomia entre teoria e prática ${ }^{(8)}$.

A RP brasileira marcou a crítica ao modelo psiquiátrico clássico e introduziu novas práticas assistenciais, pautadas no modelo psicossocial, o que impulsionou grandes transformações no cuidado de enfermagem às pessoas com transtorno mental ${ }^{(15)}$. Essa mudança de modelo tornou-se forte argumento a favor da necessidade de se repensar o ensino de enfermagem psiquiátrica, que enfatiza a atenção à saúde de diversos públicos, porém sem especificar a faixa etária a ser considerada, o que corrobora a inexistência de relatos na literatura de instituições de ensino de enfermagem que abordam a especialidade do cuidado em saúde mental infantil e juvenil em sua grade curricular. Portanto, a realidade de ensino não condiz com as demandas de assistência dos novos dispositivos de atenção à saúde mental ${ }^{(2,15)}$.

Consequentemente, os profissionais de enfermagem não demarcam com clareza qual seu trabalho nos CAPSij. A falta de reflexão, consequência de um ensino alienado, fragmenta o papel da equipe de enfermagem, fazendo com que se ocupem de trabalho que não compete exclusivamente à sua profissão, o que pode tornar sua identidade profissional distorcida ${ }^{(6)}$.

Tal distorção também é encontrada quando os profissionais de enfermagem identificam que seu trabalho não 
é sempre desenvolvido no CAPSij, sendo reconhecido por eles somente em sua dimensão prática, a partir de técnicas, o que corrobora a ideia de que o trabalho é alienado, pois acontece quando há "perda da função reflexiva através da dominação técnica, transformando o pensamento em um processo automático e instrumentalizado"(16).

Outro aspecto que demarca o não saber da equipe de enfermagem acontece quando restringem sua atuação à medida que se alienam do lugar de quem cuida e reconhecem a terapêutica como trabalho do outro. Esse recorte da alienação emerge da divisão social e técnica do trabalho, e reproduz relações ideológicas e políticas das classes sociais que reforçam a desigualdade social e a diferença entre o proprietário do saber e o proprietário da força de trabalho, demarcando uma relação de dominação ${ }^{(6)}$.

A equipe de enfermagem denomina outros profissionais da equipe como terapeutas e autoriza sua atuação em detrimento da ação própria do núcleo da enfermagem. $\mathrm{Na}$ medida em que o trabalho não é bem definido para si, os demais profissionais também não o reconhecem, resultando na atribuição de atividades simplistas e que podem ser executadas por qualquer profissional ${ }^{(6)}$, o que nos locais do estudo é possível observar a partir da criação da função de "coterapeuta”. Uma consequência é o afastamento desse profissional do lugar de agente terapêutico, tornando-o subordinado ao saber da equipe multiprofissional, o que pode romper com o desenvolvimento da clínica da enfermagem psiquiátrica ${ }^{(6)}$.

Portanto, mesmo com a existência de teorias de enfermagem que direcionam o cuidado na saúde mental, como a teoria da relação interpessoal, os profissionais de enfermagem ainda subordinam seu trabalho ao saber de outros profissionais da equipe.

Ao se considerar o trabalho em equipe nos CAPS, é possível observar que os profissionais de enfermagem são convocados pela equipe a prestar um cuidado próximo ao modelo assistencial caracterizado por garantir a higiene, autocuidado e medicação ${ }^{(17)}$.

A alienação também está presente quando há subordinação à normas de instituições externas. Isso pois o trabalho, enquanto alienado, é a forma por meio da qual o indivíduo perde-se de si mesmo e aliena-se de sua própria essência, visto que o resultado de seu trabalho é para o outro e nunca para si mesmo ${ }^{(18)}$. Como resultado, os profissionais realizam várias ações pois são uma demanda do Conselho Regional de Enfermagem, como a existência e manutenção de um posto de enfermagem e a obrigação de todos os usuários possuírem $\mathrm{SAE}$.

Porém, se por um lado identificou-se na prática de enfermagem na saúde mental infantil e juvenil um não saber pautado na falta de preparo formal e alienação, por outro lado é possível identificar um saber pautado principalmente no $\mathrm{PE}$ estruturado pela SAE.

O PE orienta ações de cuidado e auxilia a equipe de enfermagem na percepção dos problemas de saúde dos indivíduos, planejando e implementando suas ações e avaliando os resultados $^{(19)}$. Torna-se central pois possibilita uma amplitude maior na avaliação do estado de saúde do paciente, favorecendo à equipe de enfermagem assumir uma posição autônoma como agente terapêutico, o que qualifica a assistência ${ }^{(7)}$.
Entende-se o PE como referencial metodológico para construção da SAE, conceituada como método dinâmico, flexível, organizado e utilizado na prática da enfermagem para orientar o trabalho do enfermeiro, contribuindo para a delimitação de seu campo próprio de conhecimentos e para sua independência em relação às outras áreas do saber ${ }^{(19)}$.

A utilização da SAE em conjunto com o PE como método de orientação do cuidado foi um achado do estudo, porém é preciso conceituar essa prática, tendo em vista que a enfermagem pode utilizar diversas teorias científicas para embasar seu trabalho. Uma alternativa é a teoria de relação interpessoal, descrita por Peplau, que considera o trabalho central da enfermagem como sendo focado na relação interpessoal entre enfermeiro-paciente e no uso da presença e empatia, mantendo foco exclusivo ao paciente com o objetivo de conhecer qual sua perspectiva sobre si mesmo e sua situação $o^{(9)}$.

Os resultados desse estudo apontam a SAE como método para realização do acolhimento, compreendido como uma "ferramenta para humanização dos serviços de saúde, com qualificação da escuta e favorecimento da construção de vínculo" (20). Aproxima-se do cuidado de enfermagem visto que "ambos envolvem o estabelecimento de relações interpessoais com o objetivo de conforto, reconhecimento do usuário como sujeito dotado de condições objetivas e subjetivas e que está inserido em um contexto de vida"(20), o que possibilita a elaboração do histórico de enfermagem, primeira etapa do $\mathrm{PE}$.

Nesse estudo, os profissionais de enfermagem abrem espaço para a relação terapêutica no momento que reconhecem a conversa como específica de seu processo de trabalho. Na saúde mental, os momentos de interação são desenvolvidos por meio da comunicação terapêutica, entendida como a construção de um relacionamento colaborativo e focado na saúde, com o objetivo de estabelecer confiança para criar uma troca significativa ${ }^{(21)}$.

A relação terapêutica também pode ser implementada durante as atividades que são realizadas nos serviços, tal como os jogos, que podem caracterizar o brincar, atividade essencial ao bem-estar físico, emocional, mental e social da criança ${ }^{(22)}$. O bem-estar, na teoria da relação interpessoal, é alcançado quando o paciente constrói adaptações por meio da nomeação, pelo profissional de enfermagem, de seus padrões de pensamentos, comportamentos e sentimentos ${ }^{(9)}$.

Contudo, a SAE realizada nos serviços revelou uma contradição, pois o local de cuidado é reconhecido na perspectiva biológica, que encontra aplicação direta quando se observa uma queixa física, o que pode anular a subjetividade do sujeito envolvida no processo. A SAE necessita transcender a prescrição de procedimentos técnicos na medida em que deve ser utilizada como instrumento para proporcionar cuidado integral ao paciente ${ }^{(19)}$.

Tradicionalmente, a equipe de enfermagem na saúde mental trabalha dentro do modelo biomédico de atendimento, concentrando sua atenção no diagnóstico, tratamento e redução de sintomas ${ }^{(23)}$. Como consequência, o local de cuidado encontra-se no corpo, o que é possível observar quando aproximam sua prática à hospitalar, afastando o cuidado do 
modelo psicossocial, que deve incluir ações emancipatórias, de acolhimento, escuta, cuidado e enfrentamento de estigmas, considerando as singularidades de cada um e visando à melhora da qualidade de vida dos pacientes ${ }^{(2,17)}$.

O manejo dos profissionais aproxima-se da avaliação comportamental para o encaminhamento de conduta. A perspectiva comportamental é uma abordagem ativa, focada no problema e que visa a reduzir o sofrimento emocional e aumentar o comportamento adaptativo em pacientes com problemas de saúde mental ${ }^{(24)}$. Os profissionais de enfermagem, ao utilizarem esse método em conjunto com o modelo de atenção psicossocial, podem desenvolver intervenções de maneira estratégica, enquadrando estas nas etapas do PE.

Quanto à avaliação, o $\mathrm{PE}$ possibilita uma amplitude maior para sua realização sobre o estado de saúde do indivíduo, pois o foco do cuidado deve ser dirigido ao reconhecimento de sua singularidade e do "significado individual da experiência do sofrimento psíquico no seu contexto social, político e cultural, não se restringindo à sintomatologia psicopatológica e ao diagnóstico psiquiátrico"(2,7).

Já os grupos terapêuticos constituem uma ferramenta importante de escuta dos relatos de experiências do grupo, por meio do respeito à "diversidade sociocultural, focados na integralidade do atendimento, na ampliação do vínculo entre profissionais de saúde e pessoas, na educação em saúde, no desenvolvimento de autonomia e na promoção do autocuidado"(25).

No entanto, os grupos terapêuticos propostos pelos profissionais pautam-se no diagnóstico psiquiátrico, o que remonta novamente à prática baseada no modelo de atenção biomédico. Há, portanto, uma tensão entre o diagnóstico médico e o de enfermagem, sendo possível observar que os profissionais de enfermagem realizam suas intervenções baseadas no saber de outra profissão.

$\mathrm{O}$ apoio no modelo biomédico para desenvolver o processo de trabalho da enfermagem também influencia a forma como esses profissionais reconhecem sua prática em situações de crise, que demandam intervenção sobre o corpo biológico. $\mathrm{O}$ trabalho da equipe de enfermagem, nestes casos, norteia-se pelo parecer técnico do Conselho Federal de Enfermagem no 427/2012, que atribui como privativo do enfermeiro, salvo em situações de urgência e emergência, o emprego de contenção mecânica, ou em conformidade com protocolos estabelecidos pelas instituições de saúde ${ }^{(26)}$.

Considerando os momentos em que há demandas clínicas, mais precisamente cuidados físicos como curativos, cuidados de higiene e verificação de sinais vitais, estes devem ser compreendidos como parte integrante do atendimento, abrindo espaço para a interação enfermeiro-paciente e a escuta terapêutica, e não como um momento técnico e automático ${ }^{(6)}$.

Ainda, os profissionais de enfermagem identificam em seu processo de trabalho funções administrativas e de supervisão da equipe, por reconhecerem sua característica de liderança do plantão. Isso reflete os polos de direção e execução do processo de trabalho da profissão, ou seja, os aspectos administrativos e assistenciais do cuidado que resultam da divisão técnica do trabalho: os enfermeiros administrando e os técnicos e auxiliares executando ${ }^{(27)}$.
A supervisão de enfermagem possibilita melhor planejamento, implementação e avaliação do cuidado, bem como a orientação do trabalho da equipe de enfermagem ${ }^{(28)}$. Quando os profissionais dos serviços se reconhecem na liderança do plantão, caracterizada pela capacidade de motivar a realização de tarefas para atingir a excelência no trabalho ${ }^{(29)}$, não se alienam a outros profissionais e sim a aspectos administrativos, não incluindo o cuidado direto ao paciente.

$\mathrm{O}$ reconhecimento de sua potência acontece quando estão em relação, tanto com a equipe para construção do caso, como na troca relacional que acontece nos atendimentos individuais e grupais. Fazem, portanto, um recorte do trabalho no modelo biomédico e avançam no modelo psicossocial quando estão em relação. Contudo, ao se referirem ao paciente como um "caso", reforçam a presença do olhar biomédico sobre o paciente, o que se desconecta do modelo de atenção psicossocial, que considera o caso como uma construção coletiva em sua articulação com o Projeto Terapêutico Singular ${ }^{(2)}$.

Dessa forma, o avanço para o trabalho de enfermagem junto ao público infantil em CAPSij acontece quando há embasamento no saber com clara delimitação do referencial teórico, que pode apoiar-se nas teorias de enfermagem, por meio do uso do PE e no reconhecimento do profissional como agente terapêutico em momentos de cuidado físico. Por meio do PE, o profissional se reconhece como agente transformador, o que permite a utilização de diagnósticos de enfermagem para elaboração do cuidado. Os momentos de atenção à saúde física, por meio de técnicas, bem como o desenvolvimento de atividades, como o brincar, constroem um espaço de abertura para a elaboração da relação e escuta terapêutica, o que possibilita o trabalho embasado na subjetividade do sujeito. Assim, o processo de trabalho de enfermagem com o público infantil e juvenil em CAPSij caminhará para o modelo de atenção psicossocial de cuidado.

Contudo, como limitação principal desse estudo é possível apontar que o processo de trabalho da equipe de enfermagem acontece em contexto histórico e social delimitado, apreendido no método de materialismo histórico e dialético, e, portanto, seus resultados podem não compreender a prática de todos os profissionais de enfermagem considerados em um contexto mais amplo. Outra limitação encontrada diz respeito às diferentes formações dos entrevistados, sendo auxiliares, técnicos ou enfermeiros, e essa diferença impacta no processo de trabalho, a exemplo da execução do PE, já que os enfermeiros podem formular e realizar todas as etapas, enquanto os técnicos e auxiliares participam apenas de sua implementação.

\section{CONCLUSÃO}

Este estudo respondeu ao objetivo de conhecer o processo de trabalho da equipe de enfermagem em CAPSij, que acontece principalmente em dois momentos. Um deles quando os profissionais reconhecem seu processo de trabalho pelo não saber pautado no descompasso entre o que aprenderam em sua formação e o que realizam no serviço, na função de coterapeuta alienado ao saber do outro, esperando apontamento de outros profissionais, e na subordinação a 
órgãos externos. O outro acontece na prática autônoma baseada no saber desenvolvido pelo $\mathrm{PE}$ por meio da SAE pela perspectiva biológica, na elaboração de atividades, situações de manejo, avaliação do comportamento, queixa da criança e atividades em grupo, na promoção de bem-estar, em situações de crise e demandas clínicas, procedimentos técnicos, aspectos administrativos, supervisão da equipe, liderança do plantão, na construção de caso, e no espaço de troca com profissionais e pacientes.
A contribuição deste estudo evidencia-se pela contradição presente no processo de trabalho da equipe de enfermagem, que pôde ser apreendida pela tensão dialética entre o embasamento da assistência a partir do diagnóstico médico, que a aproxima da perspectiva biológica, e o modelo psicossocial do cuidado pela construção da relação terapêutica. Essa tensão pode ser efeito do trânsito que o profissional de enfermagem realiza entre o campo da saúde mental e o núcleo de enfermagem psiquiátrica.

\section{RESUMO}

Objetivo: Conhecer o processo de trabalho da equipe de enfermagem em Centros de Atenção Psicossocial Infanto-Juvenil de um município do estado de São Paulo. Método: Estudo qualitativo, descritivo e exploratório, embasado na abordagem do materialismo histórico e dialético, realizado com a equipe de enfermagem por meio de observação participante e entrevistas semiestruturadas. Resultados: Participaram 11 sujeitos da equipe de enfermagem. O processo de trabalho da equipe de enfermagem emerge da tensão dialética de duas categorias: o não saber, pautado na alienação do processo de trabalho de enfermagem e na subordinação ao saber da equipe multiprofissional, e o saber pautado na clínica de enfermagem delineada entre as perspectivas biológica e relacional. Conclusão: $\mathrm{O}$ processo de trabalho da equipe de enfermagem acontece quando pautado em um não saber pela alienação ao saber do outro, e em um saber por meio da implementação do Processo de Enfermagem pela Sistematização da Assistência de Enfermagem na perspectiva biológica, considerando aspectos relacionais e administrativos, o que se configura como uma contradição.

\section{DESCRITORES}

Enfermagem Psiquiátrica; Processo de Enfermagem; Trabalho; Criança; Saúde Mental.

\section{RESUMEN}

Objetivo: Conocer el proceso de trabajo del equipo de enfermería en Centros de Acompañamiento Psicosocial Infantil y Juvenil de un municipio de la provincia de São Paulo. Método: Estudio cualitativo, descriptivo y exploratorio, basado en el abordaje del materialismo histórico y dialéctico, realizado con el equipo de enfermería por medio de observación participante y entrevistas semiestructuradas. Resultados: Participaron 11 miembros del equipo de enfermería. Su proceso de trabajo surge de la tensión dialéctica de dos clases: el no saber, basado en la alienación del proceso de trabajo de enfermería y la subordinación al saber del equipo multiprofesional, y al saber basado en la clínica de enfermería diseñada entre la perspectiva biológica y relacional. Conclusión: El proceso de trabajo del equipo de enfermería ocurre cuando basado en un "no saber" por la alienación al saber de uno, y en un saber a través de la implementación del Proceso de Enfermería por la Sistematización del Acompañamiento de Enfermería en la perspectiva biológica, considerando aspectos de relación y administración, lo que se define como una contradicción.

\section{DESCRIPTORES}

Enfermería Psiquiátrica; Proceso de Enfermería; Trabajo; Niño; Salud Mental.

\section{REFERÊNCIAS}

1. Amarante P, Nunes MO. Psychiatric reform in the SUS and the struggle for a society without asylums. Cienc Saúde Coletiva. 2018;23(6):206774. https://doi.org/10.1590/1413-81232018236.07082018

2. Brasil. Ministério da Saúde, Secretaria de Atenção à Saúde, Conselho Nacional do Ministério Público. Atenção psicossocial a crianças e adolescentes no SUS: tecendo redes para garantir direitos. Brasília, DF: Ministério da Saúde; 2014.

3. Delfini PS, Bastos IT, Reis AO. [Family odysseys: the search for infant mental health care]. Cad Saúde Pública. 2017;33(12):e00145816. Portuguese. https://doi.org/10.1590/0102-311x00145816

4. Wieczorkievicz AM, Raissa CM, Magro IN, Mazon LM, Sprotte NL. Contribuições da enfermagem brasileira na saúde mental infantil: Uma pesquisa de revisão. Cad Bras Saúde Mental [Internet]. 2016 [cited 2020 Apr 15];8(18):xx. Available from: https://periodicos.ufsc. br/index.php/cbsm/article/view/69435

5. Brasil. Ministério da Educação. Resolução CNE/CES n. 3, de 7 de novembro de 2001. Institui diretrizes curriculares nacionais do curso de graduação em enfermagem. Diário Oficial União, Brasília, DF, 2001 Nov 9;Seçtion 1, p. 37.

6. Nicacio TR, Toledo VP, Garcia AP. From alienation to the nursing clinic: care of patients with psychiatric comorbidity. Rev Bras Enferm. 2018;71(suppl 5):2229-36. https://doi.org/10.1590/0034-7167-2017-0930

7. Garcia AP, Freitas MI, Lamas JL, Toledo VP. Nursing process in mental health: an integrative literature review. Rev Bras Enferm. 2017;70(1):220-30. https://doi.org/10.1590/0034-7167-2016-0031

8. Santos SV, Ribeiro ME, Motta AL, Silva L], Resck ZM, Terra FS. [Building knowledge in nursing: a reflective theoretical and methodological approach for nurses training]. Rev Enferm UFPE On Line. 2016;10(1):172-8. Portuguese https://doi.org/10.5205/reuol.8423-73529-1-RV1001201622

9. Delaney KR, Shattell M, Johnson ME. Capturing the interpersonal process of psychiatric nurses: a model for engagement. Arch Psychiatr Nurs. 2017;31(6):634-40. https://doi.org/10.1016/j.apnu.2017.08.003

10. Egry EY, Fonseca RM, Oliveira MA, Bertolozzi MR. Nursing in Collective Health: reinterpretation of objective reality by the praxis action. Rev Bras Enferm. 2018;71(suppl 1):710-5. https://doi.org/10.1590/0034-7167-2017-0677

11. Amoras JA, Sales AP, Sampaio AT, Machado RM, Duarte SJ. Historical and dialectical materialism in nursing care: integrative review. Rev Enferm UFPE. Online. 2016;10(4):1307-14. https://doi.org/10.5205/1981-8963-v10i4a11118p1307-1314-2016

12. Moser A, Korstjens I. Series: Practical guidance to qualitative research. Part 3: Sampling, data collection and analysis. Eur J Gen Pract. 2018;24(1):9-18. https://doi.org/10.1080/13814788.2017.1375091 
13. McGrath C, Palmgren PJ, Liljedahl M. Twelve tips for conducting qualitative research interviews. Med Teach. 2019;41(9):1002-6. https:// doi.org/10.1080/0142159X.2018.1497149

14. Crowe $M$, Inder $M$, Porter R. Conducting qualitative research in mental health: thematic and content analyses. Aust N Z J Psychiatry. 2015;49(7):616-23. https://doi.org/10.1177/0004867415582053

15. Vargas D, Maciel ME, Bittencourt MN, Lenate JS, Pereira CF. Teaching psychiatric and mental health nursing in Brazil: curricular analysis of the undergraduation course. Texto Contexto Enferm. 2018;27(2):e2610016. https://doi.org/10.1590/0104-070720180002610016

16. Costa VH. Estranho, alienação e inquietante em dialética do esclarecimento: uma antropologia entre Freud e Marx. Sapere Aude [Internet]. 2015 [cited 2020 may 15];6(11):149-65. Available from: http://periodicos.pucminas.br/index.php/SapereAude/article/view/10022

17. Oliveira RM, Alves M, Porto IS, Cavalcanti PC. The psychiatrical nursing clinic and its new care technologies. J Res Fundam Care Online. 2016;8(1):3922-34. https://doi.org/10.9789/2175-5361.2016.v8i1.3922-3934

18. Pacheco SC. O trabalho e a alienação na filosofia de Karl Marx. Rev Acad Digital [Internet]. 2018 [cited 2020 May 15];(1):85-106. Available from: http://souzaeadrevistaacademicadigital.faculdadesouza.com/uploads/revista/2018/08/souza-ead-revista-academicadigital-1535403093.pdf $\#$ page $=85$

19. Benedet SA, Gelbcke FL, Amante LN, Padilha MIS, Pires DP. [Nursing process: systematization of the nursing care instrument in the perception of nurses]. J Res Fundam Care Online. 2016 ;8(3):4780-8. Portuguese. https://doi.org 10.9789/21755361. 2016.v8i2.4780-4788

20. Costa PC, Garcia AP, Toledo VP. Welcoming and nursing care: a phenomenological study. Texto Contexto Enferm. 2016;25(1):e4550015. https://doi.org/10.1590/0104-07072016004550014

21. Martin CT, Chanda N. Mental health clinical simulation: therapeutic communication. Clin Simul Nurs. 2016;12(6):209-14. https://doi. org/10.1016/j.ecns.2016.02.007

22. Pustilnik S. Adapting well-Being into outpatient child psychiatry. Child Adolesc Psychiatr Clin N Am. 2019;28(2):221-35. https://doi. org/10.1016/j.chc.2018.11.006

23. Doyle L, Ellilä H, Jormfeldt H, Lahti M, Higgins A, Keogh B, et al. Preparing master-level mental health nurses to work within a wellness paradigm: findings from the eMenthe project. Int J Ment Health Nurs. 2018;27(2):823-32. https://doi.org/10.1111/inm.12370

24. Wenzel A. Basic strategies of cognitive behavioral therapy. Psychiatr Clin North Am. 2017;40(4):597-609. https://doi.org/10.1016/j. psc.2017.07.001

25. Alves MS, Araújo MC, Nascimento MP, Ribeiro FC, Rebouças PT, Santos TA, et al. Therapeutic group with seniors about self-care in chronic diseases. J Health Sci. 2016;18(1):48-51. https://doi.org/10.17921/2447-8938.2016v18n1p52-55

26. Conselho Federal de Enfermagem. Resolução Cofen n. 427, de 15 de fevereiro de 2012. Normatiza os procedimentos da enfermagem no emprego de contenção mecânica de pacientes. Brasília, DF: Conselho Federal de Enfermagem; 2012.

27. Leal JA, Melo CM. The nurses' work process in different countries: an integrative review. Rev Bras Enferm. 2018;71(2):413-23. https://doi. org/10.1590/0034-7167-2016-0468

28. Chaves LD, Mininel VA, Silva JA, Alves LR, Silva MF, Camelo SH. Nursing supervision for care comprehensiveness. Rev Bras Enferm. 2017;70(5):1106-11. https://doi.org/10.1590/0034-7167-2016-0491

29. Faria CC, Santos MC, Luz NC, Pereira LF, Lima RS, Haddad JG. How leader nurse communicates in a hospital: an analysis of discursive practices. J Res Fundam Care Online. 2017;9(1):152-8. https://doi.org/10.9789/2175-5361.2017.v9i1.152-158 